\title{
MicroRNA dysregulation in rhabdomyosarcoma: a new player enters the game
}

\author{
Zheng Li*, Xin Yu*, Jianxiong Shen*, Yang Liu*, Matthew T. V. Chan† and William K. K. Wu†† \\ *Department of Orthopaedic Surgery, Peking Union Medical College Hospital, Chinese Academy of Medical Sciences and Peking Union Medical \\ College, Beijing 100007, China, †Department of Anaesthesia and Intensive Care, The Chinese University of Hong Kong, Hong Kong 999077, \\ China and $\ddagger$ State-Key Laboratory of Digestive Diseases, LKS Institute of Health Sciences, The Chinese University of Hong Kong, Hong Kong \\ 999077, China
}

Received 7 March 2015; revision accepted 1 May 2015

\begin{abstract}
Rhabdomyosarcoma (RMS) is the most common of the soft tissue sarcomas with resultant high morbidity, frequently occuring in paediatric patients and young adults. While the molecular basis of RMS has received considerable attention, exact mechanisms underlying its development and metastasis remain unclear. MicroRNAs (miRNAs) are endogenously expressed small non-coding RNAs that negatively regulate gene expression via translational inhibition or mRNA degradation. Deregulated expression of miRNA has been implicated in initiation, progression, and metastasis of RMS. miRNAs have emerged as key regulators of several physiological and pathophysiological processes and have opened new avenues for diagnosis and treatment of RMS. This review summarizes deregulation and functional roles of miRNAs in RMS and their potential applications for diagnosis, prognosis and treatment of this malignancy. As a rapidly evolving field in basic and translational medicine, it is hopeful that miRNA research will ultimately improve management of RMS.
\end{abstract}

\section{Introduction}

Rhabdomyosarcoma (RMS) is the most common of the soft tissue sarcomas and frequently occurs in paediatric patients and young adults $(1,2)$. The current histological classification system categorizes it as either embryonal (ERMS) or alveolar (ARMS) type, which differ in body

Correspondence: J. Shen, Department of Orthopaedic Surgery, Peking Union Medical College Hospital, Chinese Academy of Medical Sciences and Peking Union Medical College, Beijing 100007, China. Tel.: +86010 69152812; Fax: +86010 69152812; E-mail: shenjianxiong@medmail.com.cn

Zheng $\mathrm{Li}$ and $\mathrm{Xin} \mathrm{Yu}$ contributed equally to this work. location, occurrence, mean patient age and prognosis (3-5). ERMS lesions, the most common type, have features of embryonic muscle, and are generally associated with favourable prognosis $(5,6)$. However, ARMS lesions consist of small, round and densely packed cells, displaying poor muscle differentiation, and tends to have unfavourable outcomes $(7,8)$. While ERMS tumours frequently have mutations in components of the RAS pathway (9), ARMS is usually associated with balanced chromosomal translocation, namely fusion of $P A X 3$ or PAX7 with FOXO1. Misregulated myoblast fusion caused by ectopic TANC1 expression could mediate pro-tumourigenic effects of PAX-FOXO1 fusion (10). PAX3-FOXO1 fusion has also been shown to up-regulate RASSF4 to inhibit the Hippo pathway tumour suppressor MST1(11). Until now, molecular mechanisms of RMS development have still not been fully elucidated $(7,12)$. In this regard, identification of crucial biomarkers can improve our understanding of RMS tumour biology and help us discover novel targets for its therapy in clinical settings (13-15).

MicroRNAs (miRNAs), a group of small non-coding RNAs ( $\sim 22$ nucleotides in length), carry out their biological functions by negatively regulating expression of target mRNAs at the post-transcriptional level, through base-pairing with their $3^{\prime}$-untranslated regions ( $3^{\prime}$-UTRs) (16-21). Increasing studies have indicated that miRNAs play important role in cell proliferation, apoptosis, invasion, migration and metabolism (22-25). Deregulation of miRNA has been detected in various types of cancer, including of the lung, breast, bladder, prostate and stomach $(21,26-29)$. To this end, miRNAs may function as oncogenes or tumour suppressor genes, depending on identities and functional importance of their target genes (30-33). Our review focuses on recent data related to miRNAs involved in development of RMS and discusses their potential to be used as diagnostic and prognostic biomarkers as well as in treatment strategies for RMS. 


\section{Deregulated miRNAs in RMS}

Numbers of expression profiling studies have shown that miRNAs are dysregulated in RMS (Table 1). Wei et al. performed parallel miRNA and mRNA expression profiling on 57 tumour xenografts and cell lines, representing 10 different paediatric solid tumours, using microarray analysis. Their data revealed that paediatric cancers, including RMS, osteosarcomas and neuroblastoma, have distinct miRNA expression profiles. In this respect, 14 miRNAs were found to be differentially expressed in RMS and neuroblastoma (34). A further study used supervised hierarchical clustering on RMS by an ANOVA with $P<0.03$, in four molecular subtypes of RMS (ERMS, PAX3-positive ARMS, PAX7positive ARMS, fusion-negative ARMS), revealing that tumours clustered according to their molecular alterations in PAX3/FOXO1, PAX7/FOXO1 or no translocation, on the basis of expression levels of 10 miRNAs. PAX-positive tumours (particularly PAX3), overexpressed all these miRNAs (35). A panel of 107 differentially expressed miRNAs also differentiated ARMS and malignant rhomboid tumours, the latter of which is one of the most aggressive and lethal malignancies in paediatric oncology (13). In particular, miR-9 was found to be overexpressed in ARMS and associated with metastatic invasion. In contrast, expression levels of miR200c were lower in ARMS than that in malignant rhomboid tumours. Using deep sequencing technology, Megiorni et al. reported that 97 miRNAs were deregulated in ARMS and ERMS samples compared to normal skeletal muscle. miR-378 family members were dramatically lower in RMS tumour tissues and cell lines (36).

miR-301 is up-regulated in RMS cell lines and primary tumour samples compared to human skeletal muscle cells (SkMC) and muscle tissue controls (7). Sarver et al. reported that miR-183 was overexpressed in RMS as well as in corresponding tumour cell lines (37) and Reichek et al. determined miRNA expression in relation to amplification of the 13q31 chromosomal region, which harbours the miR-17-92 cluster (miR-17, miR-19a, miR19b, miR-20a and miR-92a), in ARMS (38). They found that in tumours with the $13 \mathrm{q} 31$ amplification, there was higher expression of five of six microRNAs within the cluster. In addition, a subset of non-amplified tumours with copy number-independent overexpression of all six microRNAs was identified. miR-29 is epigenetically silenced in RMS cells and primary tumours that are poorly differentiated (3). miR-27a and miR-26a were down-regulated in all RMS tissues compared to muscle tissues, suggesting their potential roles as tumour suppressors in RMS (7); Taulli et al. also showed that miR-1 and miR206 expression was lower in primary RMS (39). Moreover, down-regulation of these two miRNAs was confirmed by Yan et al. in RMS tissues and cell lines (40). Missiaglia et al. reported that muscle-specific miRNAs, including miR-1, miR-206, miR-133a and miR-133b, were lower in RMSs compared to skeletal muscle (41). Rao et al. found that expression of miR-1 and miR-133a were low in representative cell lines from ERMS and ARMS (42). Diao et al. reported that miR-203 was frequently down-regulated by promoter hypermethylation in both RMS cell lines and RMS biopsies and could be reactivated by DNA-demethylating agents (43).

It is noteworthy that only small numbers of deregulated miRNAs were shared between different studies and several miRNAs even exhibited discordant expression patterns. These discrepancies are probably due to quality of clinical samples, indistinctive changes, specificity of profiling platforms, different protocols for sample collection and processing, preceding cytotoxic treatments, tumour heterogeneity and underestimated hypoxia and infection. Thus, it is important to re-evaluate current strategies in miRNA profiling and be cautious concerning interpretation of existing signatures.

\section{Mechanisms of miRNA deregulation in RMS}

Expression of miRNA is regulated in ways similar to those of other coding genes. Recent pieces of work have pro-

Table 1. miRNA expression profiles in rhabdomyosarcoma (RMS)

\begin{tabular}{|c|c|c|c|c|c|}
\hline No. & Sample & Tumour-specific & Up-regulated & Down-regulated & References \\
\hline 1 & $\begin{array}{l}\text { Xenograft } \\
\text { Cell lines } \\
\text { Rhabdomyosarcoma } \\
\text { /neuroblastoma }\end{array}$ & 14 miRNA & & & (34) \\
\hline 2 & Primary RMS different subtypes of RMS & 10 miRNA & & & (35) \\
\hline 3 & MRT/RMA & 107 miRNA & 46 miRNA & 61 miRNA & (13) \\
\hline 4 & ARMS, ERMS/NSM & 97 miRNA & 18 miRNA & 79 miRNA & (36) \\
\hline
\end{tabular}

RMA, Alveolar rhabdomyosarcoma; MRT, malignant rhabdoid tumour; ARMS, alveolar rhabdomyosarcoma; ERMS, embryonal rhabdomyosarcoma; NSM, normal skeletal muscle. 
vided new insights to explain miRNA deregulation in RMS, including epigenetic alteration and deregulated transcription. As mentioned above, miR-29 can be epigenetically silenced by activated nuclear factor- $\kappa \mathrm{B}(\mathrm{NF}-\kappa \mathrm{B})-$ Ying Yang 1 (YY1) pathway in RMS cells and primary tumours (3). A further study has demonstrated that activation of haeme oxygenase-1 (a cytoprotective enzyme induced in response to oxidative stress), in C2C12 cells, reduced abundance of miR-1, miR-133a, miR-133b and miR-206, which was accompanied by augmented production of SDF-1 and miR-146a (44). miR-203 was frequently down-regulated by promoter hypermethylation but could be reactivated by 5 -aza- $2^{\prime}$-deoxycytidine treatment (43). Megiorni et al. also showed that DNA demethylation by 5-aza-2'-deoxycytidine was able to up-regulate miR-378a$3 \mathrm{p}$ (36). Sun et al. reported that TGF- $\beta 1$ exerted its function by suppressing miR-450b-5p in RMS (45).

Biogenesis of miRNAs can also be altered in RMS. Dicer is an endoribonuclease involved in processing premiRNA into mature miRNA. Somatic Dicer 1 mutations have been found in $3.8 \%$ of sporadic ERMS (46).

\section{Biological functions of deregulated miRNAs in RMS}

As increasing deregulated miRNAs have been detected, further understanding of their functional roles, especially their interactions with tumour suppressor genes, oncogenes or other cancer-related genes, it is critical for us to elucidate the molecular tumourigenesis of RMS (Table 2).

Several deregulated miRNAs have been implicated in differentiation of muscle cells. For instance, reconstitution of miR-29 in murine RMS, inhibited tumour growth and stimulated differentiation by targeting YY1. As noted above, miR-29 could be silenced by YY1, thus forming molecular circuitry that involves mutual inhibition between YY1 and miR-29 (3). Overexpression of miR203 in RMS cells inhibited their migration and proliferation and promoted terminal myogenic differentiation. Mechanistically, miR-203 has been found to exert its tumour-suppressive effect by directly targeting p63 and leukaemia inhibitory factor receptor in RMS cells, which promotes myogenic differentiation by inhibiting Notch and Janus kinase 1 (JAK1)/signal transducer, and activator of transcription 1/3 (STAT1/3) pathways, supporting the role of miR-203 as a tumour suppressor in RMS (43). A further study indicates that re-expression of miR-378a3 p causes significant changes in apoptosis, cell migration, cytoskeleton organization as well as modulation of muscle markers MyoD1, MyoR, desmin and the myosin heavy chain (36). In addition, DNA demethylation by 5aza- $2^{\prime}$-deoxycytidine has been found to be able to up-regulate miR-378a-3p with concomitant induction of apoptosis, decrease in cell viability and cell cycle arrest in G2phase. Morphology and expression of the myosin heavy chain in RMS cells treated with 5-aza-2'-deoxycytidine also changed. miR-378a-3p overexpression in one RMS cell line reduced IGF1R expression and phosphorylatedAkt protein levels (36). Taulli et al. found that reexpression of miR-206 in RMS cells promoted myogenic differentiation and blocked tumour growth in xenografted mice. This was indicated by switching the global mRNA expression profile to one that resembled mature muscle. c-Met, a tyrosine-kinase receptor over-expressed in RMS,

Table 2. Functional characterization of deregulated miRNAs in rhabdomyosarcoma (RMS)

\begin{tabular}{|c|c|c|c|c|}
\hline Name & $\begin{array}{l}\text { Up- or down-regulation } \\
\text { (Rhabdomyosarcoma/Normal) }\end{array}$ & Target gene & Role & Reference \\
\hline miR-29 & Down & YY1, PAX3, CCND2 & Tumour suppressor & $(3,44)$ \\
\hline miR-301 & Up & & oncogene & (7) \\
\hline miR-27a & Down & & Tumour suppressor & (7) \\
\hline miR-26a & Down & & Tumour suppressor & (7) \\
\hline miR-1 & Down & c-Met, PAX3, CCND2 & Tumour suppressor & $(37-40,44)$ \\
\hline miR-206 & Down & c-Met, PAX3, CCND2 & Tumour suppressor & $(37-39,44)$ \\
\hline miR-133a & Down & TPM4 & Tumour suppressor & $(39,40)$ \\
\hline miR-133b & Down & & Tumour suppressor & (39) \\
\hline $\operatorname{miR}-183$ & Up & $\begin{array}{l}\text { EGR1 } \\
\text { PTEN }\end{array}$ & oncogene & (41) \\
\hline $\begin{array}{l}\text { miR-17, miR-19a, miR-19b, } \\
\text { miR-20a, miR-92a }\end{array}$ & Up & & & $(42)$ \\
\hline miR-203 & Down & p63 & Tumour suppressor & (43) \\
\hline miR-378a-3p & Down & IGF1R & Tumour suppressor & (36) \\
\hline $\operatorname{miR}-485-3 p$ & Up & NF-YB & oncogene & (45) \\
\hline $\operatorname{miR}-450 b-5 p$ & Down & $\begin{array}{l}\text { ENOX2 } \\
\text { PAX9 }\end{array}$ & Tumour suppressor & (47) \\
\hline miR-214 & Down & N-ras & Tumour suppressor & (49) \\
\hline
\end{tabular}


was down-regulated in murine satellite cells by miR-206 at the onset of normal myogenesis (39). In addition, Wang et al. showed that reconstitution of miR-29 in RMS in mice inhibited tumour growth and stimulated differentiation, suggesting that miR-29 acted as a tumour suppressor through its promyogenic function (3). Sun et al. reported that miR-450b-5p arrested expansion of RMS and promoted expression of MyoD, a protein that plays a major role in regulating muscle differentiation. Utilizing a bioinformatics approach, miR-450b-5p target mRNAs were identified. Among these candidates, expression of ENOX2 and PAX9 only were augmented by miR450b-5p knock-down (45). A further recent study showed that overexpression of miR-214 inhibited RMS tumour growth, induced myogenic differentiation and apoptosis, as well as suppressed colony formation and xenograft tumourigenesis. N-Ras is a conserved target of miR-214 and its expression is up-regulated in human RMS tissues (47). Rao et al. demonstrated that miR-1 and miR-133a exerted cytostatic effects in an ERMS cell line, suggesting a tumour suppressor-like role for these myogenic miRNAs. Transcriptional profiling of cells transfected with miR-1 and miR-133a revealed that miR-1 but not miR133 a exerted strong promyogenic influence on poorly differentiated tumour cells. mRNA targets (such as ADAR, ANXA2, ZFP36L1 for miR-1; TNFRSF10B, CORO1C and LASS2 for miR-133a) of miR-1 and miR-133a were also up-regulated in RMS, suggesting a causative role for these miRNAs in RMS development (42).

Cell cycle progression is another key cellular process deregulated by miRNAs in RMS. In this regard, miR-1, miR-206 and miR-29 have been reported to regulate expression of CCND2, a cell cycle gene. miR-29 also targeted a further cell cycle regulator E2F7. To this end, ectopic expression of miR-29 down-regulated expression of these cell cycle genes and induced partial G1 arrest, leading to reduced cell proliferation. These data support tumour suppressor roles for miR-1, miR-206 and miR29 in RMS. A previous study has also demonstrated that transient transfection of miR-1 and miR-206 into cultured RMS RD cells led to a significant decrease in cell proliferation and migration. By bioinformatic analysis combined with Western blotting, putative binding sites for miR-1 and miR-206 within the $3^{\prime}$-UTR of human cMet mRNA have been identified and down-regulation of c-Met protein by miR-1 and miR-206 was confirmed (40), suggesting that miR-1 and miR-206 might exert tumour suppressor activity by targeting c-Met. Importantly, up-regulation of c-Met was confirmed in tissue samples of human RMS, with levels inversely correlated to $\mathrm{miR}-1$ and miR-206 expression. In vivo, miR-1- or miR-206-expressing tumour cells had remarkable growth delay (40). BAF53a, a subunit of the SWI/SNF chro- matin remodelling complex, is also the direct target of miR-206(48). Li et al. showed that ectopic expression of miR-1 and miR-206 in JR1, an ERMS cell line, down-regulated PAX3, whereas overexpression of these two miRNAs in Rh30, an ARMS cell line, did not have any effect on PAX3 protein levels (49). In ARMS, PAX3 forms a fusion transcript with FOXO1 and resultant loss of PAX3 $3^{\prime}$ UTR in the fusion transcript represents an oncogenic mechanism to evade miRNAmediated regulation of $\mathrm{PAX} 3$. Chen et al. demonstrated that restored expression of miR-485-3p in human lymphoblastic leukaemia cells (CEM) could reduce expression of NF-YB accompanied by corresponding upregulation of DNA topoisomerase II $\alpha$ and increased sensitivity to DNA topoisomerase II inhibitors. Importantly, results from CEM cells were replicated in both drugsensitive and -resistant human RMS Rh30 cells (50). Sarver et al. demonstrated deregulation of a miRNA network composed of miR-183-EGR1-PTEN in synovial sarcoma, RMS and colon cancer cell lines. Integrated miRNA- and mRNA-based genomic analyses indicated that miR-183 was a key contributor to cell migration in these tumour types and such regulation occurred via EGR1-based mechanism. miR-183 has a potential oncogenic role through regulation of two tumour suppressor genes, EGR1 and PTEN, and deregulation of this fundamental miRNA regulatory network may be central to many tumour types, including RMS (37).

\section{Prognostic use of miRNAs and other clinical implications}

Efforts have been made to predict disease outcome and response to treatment in relation to miRNA expression. Missiaglia et al. demonstrated that low miR-206 expression was an independent predictor of shorter overall survival of patients with metastatic ERMS and ARMS cases without PAX3/7-FOXO1 fusion. Low miR-206 expression also correlated with high Societe Internationale D'oncologie Pediatrique (SIOP) stage and presence of metastases at diagnosis. Low miR-206 is linked to aberrant activation of mitogen-activated protein kinase (MAPK) and NF-кB pathways, whereas high miR-206 expression induced genes linked to muscle differentiation (41). In addition, Miyachi et al. reported that serum levels of muscle-specific miRNAs (miR-1, miR-133a, miR-133b and miR-206) were higher in patients with RMS than in patients with non-RMS (51). However, the mechanisms by which serum levels of these miRNAs were elevated remained unclear as these miRNAs are down-regulated in RMS tissues. Normalized serum miR206 expression levels can be used to differentiate between RMS and non-RMS, with sensitivity of 1.0 and 
specificity of 0.913. In ARMS, increased expression of miR-17-92 cluster has been found, with marked preference in PAX7-FOXO1-positive cases. In clinical analyses, poorer outcomes were associated with increased expression of this cluster in 13q31-amplified cases compared to non-amplified cases (38). There was also improved outcome in 13q31-amplified cases with lower expression of these miRNAs. Thus, 13q31 amplification and expression of the miR-17-92 cluster provide novel markers for identifying differential prognostic subsets in ARMS (38). These results suggest that miRNAs are useful biomarkers for early diagnosis and prognosis of RMS. It is expected that incorporation of miRNA into current panels of biomarkers will enhance sensitivity and specificity of non-invasive diagnostic and prognostic tests for RMS.

\section{Conclusions and future perspectives}

miRNAs, presenting an endogenous form of RNA interference, are now considered to be potential therapeutic targets and new biomarkers for RMS (8). It is also welldemonstrated that pathogenic roles of deregulated miRNAs have been extensively studied (52). Various miRNA profiling studies have shown that RMS patients display unique miRNA signatures, which are associated with RMS development or metastasis $(13,36)$. Moreover, miRNAs can play crucial roles in cell proliferation, invasion, apoptosis, migration and metabolism of RMS cells $(42,43,47)$. Their potential to act as tumour suppressors or oncogenes under external stimulation also makes them prominent targets for therapeutic intervention. In addition, although miRNA-based therapy is not currently used in the clinic, its innovative applications are growing in various fields. However, the list of targetable miRNAs in RMS is far from complete and their therapeutic efficacies remain unclear. Further analyses and new technologies in miRNA research will definitely shed new light on pathogenesis of RMS. Consequently, analysing miRNA profiles and their signalling pathways will offer deeper insights into the treatment options for RMS.

\section{Acknowledgements}

This work was supported by grants from the National Natural Science Foundation of China (NSFC) (Grant numbers: 81401847,81272053 and 81330044$)$.

\section{Conflict of interest}

The authors declare no conflict of interest.

\section{References}

1 Keller C, Guttridge DC (2013) Mechanisms of impaired differentiation in rhabdomyosarcoma. FEBS J. 280, 4323-4334.

2 Williamson D, Selfe J, Gordon T, Lu YJ, Pritchard-Jones K, Murai $\mathrm{K}$ et al. (2007) Role for amplification and expression of glypican-5 in rhabdomyosarcoma. Cancer Res. 67, 57-65.

3 Wang H, Garzon R, Sun H, Ladner KJ, Singh R, Dahlman J et al. (2008) NF-kappaB-YY1-miR-29 regulatory circuitry in skeletal myogenesis and rhabdomyosarcoma. Cancer Cell 14, 369-381.

4 Ianzano ML, Croci S, Nicoletti G, Palladini A, Landuzzi L, Grosso $\mathrm{V}$ et al. (2014) Tumor suppressor genes promote rhabdomyosarcoma progression in 553 heterozygous, HER-2/neu transgenic mice. Oncotarget 5, 108-119.

5 Srivastava RK, Kaylani SZ, Edrees N, Li C, Talwelkar SS, Xu J et al. (2014) GLI inhibitor GANT-61 diminishes embryonal and alveolar rhabdomyosarcoma growth by inhibiting Shh/AKT-mTOR axis. Oncotarget 5, 12151-12165.

6 Jothi M, Mal AK (2012) Too much AKT turns PAX3-FKHR dead: a prospect of novel therapeutic strategy for alveolar rhabdomyosarcoma. Oncotarget 3, 1064-1065.

7 Ciarapica R, Russo G, Verginelli F, Raimondi L, Donfrancesco A, Rota R et al. (2009) Deregulated expression of miR-26a and Ezh2 in rhabdomyosarcoma. Cell Cycle 8, 172-175.

8 Ciesla M, Dulak J, Jozkowicz A (2014) MicroRNAs and epigenetic mechanisms of rhabdomyosarcoma development. Int. J. Biochem. Cell Biol. 53, 482-492.

9 Martinelli S, McDowell HP, Vigne SD, Kokai G, Uccini S, Tartaglia M et al. (2009) RAS signaling dysregulation in human embryonal Rhabdomyosarcoma. Genes Chromosom. Cancer 48, 975-982.

10 Avirneni-Vadlamudi U, Galindo KA, Endicott TR, Paulson V, Cameron S, Galindo RL. (2012) Drosophila and mammalian models uncover a role for the myoblast fusion gene TANC1 in rhabdomyosarcoma. J. Clin. Invest. 122, 403-407.

11 Crose LE, Galindo KA, Kephart JG, Chen C, Fitamant J, Bardeesy $\mathrm{N}$ et al. (2014) Alveolar rhabdomyosarcoma-associated PAX3FOXO1 promotes tumorigenesis via Hippo pathway suppression. $J$. Clin. Invest. 124, 285-296.

12 Bian L, Wang Y, Liu Q, Xia J, Long JE (2015) Prediction of signaling pathways involved in enterovirus 71 infection by algorithm analysis based on miRNA profiles and their target genes. Arch. Virol. 160, 173-182.

13 Armeanu-Ebinger S, Herrmann D, Bonin M, Leuschner I, Warmann SW, Fuchs J et al. (2012) Differential expression of miRNAs in rhabdomyosarcoma and malignant rhabdoid tumor. Exp. Cell Res. 318, 2567-2577.

14 Seitz G, Warmann SW, Fuchs J, Heitmann H, Mahrt J, Busse AC et al. (2008) Imaging of cell trafficking and metastases of paediatric rhabdomyosarcoma. Cell Prolif. 41, 365-374.

15 He F, Yao H, Wang J, Xiao Z, Xin L, Liu Z et al. (2015) Coxsackievirus B3 engineered to contain MicroRNA targets for muscle-specific MicroRNAs displays attenuated cardiotropic virulence in mice. J. Virol. 89, 908-916.

16 Fei B, Wu H (2013) MiR-378 inhibits progression of human gastric cancer MGC-803 cells by targeting MAPK1 in vitro. Oncol. Res. 20, 557-564.

17 Wu W, He X, Kong J, Ye B (2012) Mir-373 affects human lung cancer cells' growth and its E-cadherin expression. Oncol. Res. 20, $163-170$.

18 Liang J, Zhang Y, Jiang G, Liu Z, Xiang W, Chen X et al. (2013) MiR-138 induces renal carcinoma cell senescence by targeting EZH2 and is downregulated in human clear cell renal cell carcinoma. Oncol. Res. 21, 83-91. 
19 Xiong X, Ren HZ, Li MH, Mei JH, Wen JF, Zheng CL et al. (2011) Down-regulated miRNA-214 induces a cell cycle G1 arrest in gastric cancer cells by up-regulating the PTEN protein. Pathol. Oncol. Res. 17, 931-937.

20 Zhang C, Liu J, Wang X, Wu R, Lin M, Laddha SV et al. (2014) MicroRNA-339-5p inhibits colorectal tumorigenesis through regulation of the MDM2/p53 signaling. Oncotarget 5, 9106-9117.

21 Yang WB, Chen PH, Hsu Ts FuTF, Su WC, Liaw H et al. (2014) Sp1-mediated microRNA-182 expression regulates lung cancer progression. Oncotarget 5, 740-753.

22 Wang K, Jia Z, Zou J, Zhang A, Wang G, Hao J et al. (2013) Analysis of hsa-miR-30a-5p expression in human gliomas. Pathol. Oncol. Res. 19, 405-411.

23 Chiang Y, Zhou X, Wang Z, Song Y, Liu Z, Zhao F et al. (2012) Expression levels of microRNA-192 and -215 in gastric carcinoma. Pathol. Oncol. Res. 18, 585-591.

24 Fei B, Wu H (2012) MiR-378 inhibits progression of human gastric cancer MGC-803 cells by targeting MAPK1 in vitro. Oncol. Res. 20, 557-564.

25 Li H, Yang BB (2012) Stress response of glioblastoma cells mediated by miR-17-5p targeting PTEN and the passenger strand miR17-3p targeting MDM2. Oncotarget 3, 1653-1668.

26 He X, Li J, Guo W, Liu W, Yu J, Song W et al. (2014) Targeting the microRNA-21/AP1 axis by 5-fluorouracil and pirarubicin in human hepatocellular carcinoma. Oncotarget 5, 6654-6669.

27 Chakravarthi BV, Pathi SS, Goswami MT, Cieslik M, Zheng H, Nallasivam S et al. (2014) The miR-124-prolyl hydroxylase P4HA1-MMP1 axis plays a critical role in prostate cancer progression. Oncotarget 5, 6654-6669.

28 Wang Z, Wang N, Liu P, Chen Q, Situ H, Xie T et al. (2014) MicroRNA-25 regulates chemoresistance-associated autophagy in breast cancer cells, a process modulated by the natural autophagy inducer isoliquiritigenin. Oncotarget 5, 7013-7726.

29 Ouchida M, Kanzaki H, Ito S, Hanafusa H, Jitsumori Y, Tamaru S et al. (2012) Novel direct targets of miR-19a identified in breast cancer cells by a quantitative proteomic approach. PLOS ONE 7, e44095.

30 Li Z, Lei H, Luo M, Wang Y, Dong L, Ma Y et al. (2015) DNA methylation downregulated mir-10b acts as a tumor suppressor in gastric cancer. Gastric Cancer 18, 43-54.

31 Yu X, Li Z, Shen J, Wu WK, Liang J, Weng X et al. (2013) MicroRNA-10b promotes nucleus pulposus cell proliferation through RhoC-Akt pathway by targeting HOXD10 in intervetebral disc degeneration. PLOS ONE 8, e83080.

32 Li Z, Yu X, Shen J, Wu WK, Chan MT (2015) MicroRNA expression and its clinical implications in Ewing's sarcoma. Cell Prolif. 48, 1-6.

$33 \mathrm{Yu} \mathrm{X,} \mathrm{Li} \mathrm{Z} \mathrm{(2014)} \mathrm{MicroRNAs} \mathrm{regulate} \mathrm{vascular} \mathrm{smooth} \mathrm{muscle}$ cell functions in atherosclerosis (review). Int. J. Mol. Med. 34, 923-933.

34 Wei JS, Johansson P, Chen QR, Song YK, Durinck S, Wen X et al. (2009) microRNA profiling identifies cancer-specific and prognostic signatures in pediatric malignancies. Clin. Cancer Res. 15, 5560-5568.

35 Gougelet A, Perez J, Pissaloux D, Besse A, Duc A, Decouvelaere AV et al. (2011) miRNA profiling: how to bypass the current difficulties in the diagnosis and treatment of Sarcomas. Sarcoma 2011, 460650.

36 Megiorni F, Cialfi S, McDowell HP, Felsani A, Camero S, Guffanti A et al. (2014) Deep Sequencing the microRNA profile in rhabdomyosarcoma reveals down-regulation of miR-378 family members. BMC Cancer 14, 880.
37 Sarver AL, Li L, Subramanian S (2010) MicroRNA miR-183 functions as an oncogene by targeting the transcription factor EGR1 and promoting tumor cell migration. Cancer Res. 70, 9570-9580.

38 Reichek JL, Duan F, Smith LM, Gustafson DM, O'Connor RS, Zhang C et al. (2011) Genomic and clinical analysis of amplification of the $13 \mathrm{q} 31$ chromosomal region in alveolar rhabdomyosarcoma: a report from the Children's Oncology Group. Clin. Cancer Res. 17, 1463-1473.

39 Taulli R, Bersani F, Foglizzo V, Linari A, Vigna E, Ladanyi M et al. (2009) The muscle-specific microRNA miR-206 blocks human rhabdomyosarcoma growth in xenotransplanted mice by promoting myogenic differentiation. J. Clin. Invest. 119, 23662378.

40 Yan D, Dong Xda E, Chen X, Wang L, Lu C, Wang J et al. (2009) MicroRNA-1/206 targets c-Met and inhibits rhabdomyosarcoma development. J. Biol. Chem. 284, 29596-29604.

41 Missiaglia E, Shepherd CJ, Patel S, Thway K, Pierron G, Pritchard-Jones K et al. (2010) MicroRNA-206 expression levels correlate with clinical behaviour of rhabdomyosarcomas. $\mathrm{Br} . \mathrm{J}$. Cancer 102, 1769-1777.

42 Rao PK, Missiaglia E, Shields L, Hyde G, Yuan B, Shepherd CJ et al. (2010) Distinct roles for miR-1 and miR-133a in the proliferation and differentiation of rhabdomyosarcoma cells. FASEB J. 24, 3427-3437.

43 Diao Y, Guo X, Jiang L, Wang G, Zhang C, Wan J et al. (2014) miR-203, a tumor suppressor frequently down-regulated by promoter hypermethylation in rhabdomyosarcoma. J. Biol. Chem. 289 , $529-539$.

44 Kozakowska M, Ciesla M, Stefanska A, Skrzypek K, Was H, Jazwa A et al. (2012) Heme oxygenase-1 inhibits myoblast differentiation by targeting myomirs. Antioxid. Redox Signal. 16, 113127.

45 Sun MM, Li JF, Guo LL, Xiao HT, Dong L, Wang F et al. (2014) TGF-beta1 suppression of microRNA-450b-5p expression: a novel mechanism for blocking myogenic differentiation of rhabdomyosarcoma. Oncogene 33, 2075-2086.

46 Doros L, Yang J, Dehner L, Rossi CT, Skiver K, Jarzembowski JA et al. (2012) DICER1 mutations in embryonal rhabdomyosarcomas from children with and without familial PPB-tumor predisposition syndrome. Pediatr. Blood Cancer 59, 558-560.

47 Huang HJ, Liu J, Hua H, Li SE, Zhao J, Yue S et al. (2014) MiR214 and N-ras regulatory loop suppresses rhabdomyosarcoma cell growth and xenograft tumorigenesis. Oncotarget 5, 2161-2175.

48 Taulli R, Foglizzo V, Morena D, Coda DM, Ala U, Bersani F et al. (2014) Failure to downregulate the BAF53a subunit of the SWI/SNF chromatin remodeling complex contributes to the differentiation block in rhabdomyosarcoma. Oncogene 33, 2354-2362.

49 Li L, Sarver AL, Alamgir S, Subramanian S (2012) Downregulation of microRNAs miR-1, -206 and -29 stabilizes PAX3 and CCND2 expression in rhabdomyosarcoma. Lab. Invest. 92, 571583.

50 Chen CF, He X, Arslan AD, Mo YY, Reinhold WC, Pommier Y et al. (2011) Novel regulation of nuclear factor-YB by miR-485-3p affects the expression of DNA topoisomerase Ilalpha and drug responsiveness. Mol. Pharmacol. 79, 735-741.

51 Miyachi M, Tsuchiya K, Yoshida H, Yagyu S, Kikuchi K, Misawa A et al. (2010) Circulating muscle-specific microRNA, miR-206, as a potential diagnostic marker for rhabdomyosarcoma. Biochem. Biophys. Res. Commun. 400, 89-93.

52 Dela Cruz F, Matushansky I (2011) MicroRNAs in chromosomal translocation-associated solid tumors: learning from sarcomas. Discov. Med. 12, 307-317. 